\title{
Effects of grape seed extract on properties of type I collagen sca- ffolds
}

\author{
Claudio Fernandes Garcia', Virgínia CA Martins' ${ }^{1}$, Ana MG Plepis ${ }^{1}$ \\ *Corresponding author: E-mail address: claudiofgarcia@usp.br
}

Abstract: To obtain a material with potential for use in tissue engineering, anionic collagen was obtained from porcine serosa (S) and bovine tendon $(\mathrm{T})$ by alkaline hydrolysis for $72 \mathrm{~h}$. Part of this collagen was mixed with water to obtain $4 \%$ (weight/weight) collagen suspension and part was solubilized in acetic acid pH 3.5 to obtain 1.5\% (w/w) gel. The suspensions were mixed with their respective gels (2:1) (suspension: gel) and grape seed extract, whose main product is proanthocyanidin, was added at concentrations of $0.03 \%$ and $0.5 \%$, thus obtaining the scaffolds SC (serosa collagen suspension and gel), TC (tendon collagen suspension and gel), SCP003 (SC with $0.03 \%$ extract), TCP003 (TC with $0.03 \%$ extract), SCP05 (SC with $0.5 \%$ extract added) and TCP05 (TC with $0.5 \%$ extract). The materials were analyzed by differential scanning calorimetry (DSC), scanning electron microscopy (SEM) and characterized by phosphate buffered saline absorption assay and in vitro biological stability assay. By DSC it is observed that the addition of $0.5 \%$ of extract increases the denaturation temperature (Td) of collagen, indicating that at this concentration the extract acts as polymer crosslinking agent. SEM shows disorganized cross-section pores in all scaffolds, not exceeding $130 \mu \mathrm{m}$. Absorption and degradation assays indicated that the addition of $0.5 \%$ addition of $0.5 \%$ extract increases the absorption of phosphate buffered saline (PBS) by the scaffolds and decreases the degradation percentage by collagenase. These results suggests that the scaffolds can be used for different applications, e.g. as hemostatic agent.

Keywords: Biopolymer; bovine tendon; porcine serosa; crosslinking.

\section{Introduction}

Topical absorbable hemostatic agents may be used to stop bleeding and retain wound exudate as they initiate platelet aggregation allowing blood coagulation. ' Different hemostatic agents have been developed and are available to assist surgeons in the treatment of bleeding. Among the various materials used are the microfibrillary collagen, bovine or porcine gelatin sponges, oxidized cellulose, gelatin sealant matrix, thrombin, etc. 1,2,3

Collagen and its derivatives are chemically attractive in wound healing, with good biological and hemostatic properties. In addition, they have biocompatible, low toxicity and biodegradable characteristics ${ }^{4}$. Its use as a hemostatic agent occurs due to its capacity for platelet aggregation and ability to initiate the cascade of blood coagulation. ${ }^{5,6}$

Collagen is the most abundant structural protein in the body of mammals. It is present in tendons, skin, blood vessels, intestines, bones and teeth and can be extracted from all these sources. The tendons are formed by dense regular connective tissue, which presents fibers arranged in a single direction giving tensile strength and low flexibility, unlike mature bone tissue, which presents collagen fibers arranged in the form of lamellae. Mucosa, muscularis, submucosa, and serosa layers form the intestine, which is composed of loose connective tissue, presenting collagen fibers without order. ${ }^{7,8,9}$

A chemical modification that can be performed with collagen is the selective hydrolysis of the carboxyamide groups of the asparagine and glutamine amino acid residues, leading to an increase of up to 134 negative charges per molecule.. ${ }^{10}$ This modification allows the production of negatively charged collagen scaffolds at $\mathrm{pH} 7.4$ with high biocompatibility and almost complete absence of chronic inflammatory response. ${ }^{11,12,13}$

Another modification that collagen may suffer is the crosslinking reaction, which increases the resistance to in vitro degradation and alters its mechanical properties. ${ }^{14}$ Proanthocyanidins are polyphenolic compounds in the category known as condensed tannins, which can be used as crosslinking agents due to the hydroxyl groups present in their structure, giving stability to hydrogen bonds and thus generating collagen structures with less biodegradability. ${ }^{15}$

Grape seed extract is a byproduct of the production of wines and sparkling wines, presenting several flavonoids such as anthocyanins, flavonols, catechins and proanthocyanidins, the most abundant. Proanthocyanidin has free radical scavenging ability, having superior antioxidant capacity than other oxidants like vitamin $C$, vitamin $E, \beta$-carotene. In addition, it has several pharmacological and biological properties, namely: cardioprotective, anti-tumor, bactericidal and anti-inflammatory. ${ }^{16}$

This study aims to obtain and characterize neutral scaffolds of anionic collagen obtained from two distinct sources (bovine tendon and porcine serosa), crosslinked with grape seed extract and having potential to be used as a hemostatic agent.

\section{Materials and methods}

\section{Materials}

Bovine tendon and porcine serosa were obtained at meat houses, and grape seed extract (Vitis sp.) was obtained from a drugstore.

Anionic collagen was obtained from two distinct sources, bovine tendon and porcine serosa, by the treatment in an aqueous alkaline solution ( $\mathrm{pH} \sim 13$ ) for $72 \mathrm{~h}$ using a protocol developed in previous studies in our laboratory. ${ }^{17}$ In short, tendon or serosa were placed in an alkaline solution containing $\mathrm{Ca}(\mathrm{OH}) 2, \mathrm{KOH}, \mathrm{NaOH}, \mathrm{Na} 2 \mathrm{SO} 4, \mathrm{CaSO} 4, \mathrm{KCl}$ and $\mathrm{NaCl}$ for $72 \mathrm{~h}$. Subsequently, the material was equilibrated for $6 \mathrm{~h}$ in a solution containing the same salts. Excess salts were removed by rinsing in 3\% (w/w) boric acid, deionized water, $0.3 \%$ (w/w) EDTA solution, at pH 11 and deionized water up to $\mathrm{pH} 7$.

Treated porcine serosa in water was ground in a blender and later its concentration was defined by lyophilization. Subsequently, it was divided in two parts, the first one was used to prepare a $4 \%$ suspension in water and the second one was solubilized in acetic acid pH 3.5 to obtain a $1 \%$ collagen gel.

Treated bovine tendon was lyophilized and grounded in a blender. It was later divided in two parts, the first of which was suspended in water to obtain a $4 \%$ suspension and the other one was solubilized in acetic acid $\mathrm{pH} 3.5$ to obtain a $1 \%$ collagen gel.

The scaffolds were obtained by mixing the suspension with the collagen gel from the same source, in a 2:1 ratio (suspension: gel) and adding

$\overline{1}$ Institute of Chemistry of São Carlos - IQSC - University of São Paulo - USP,Brazil. 
or not grape seed extract, which was previously solubilized in $20 \mathrm{~mL}$ of $50 \%$ hydroethanolic.

Concentrations of $0.03 \%$ and $0.5 \%$ of grape seed extract were chosen based on previous studies in our laboratory.
The mixtures were placed in Teflon ${ }^{\circledR}$ molds and then lyophilized. They were neutralized using ammonia vapor for a period of $24 \mathrm{~h}$ and subsequently left under constant air flow to remove excess ammonia for $72 \mathrm{~h}$. Scaffolds were nominated as shown in Table 1.

\begin{tabular}{cc}
\hline Scaffold & Composition \\
\hline SC & serosa suspension and its gel \\
SCP003 & serosa suspension and its gel + grape seed extract $0.03 \%(\mathrm{w} / \mathrm{w})$ \\
SCP05 & serosa suspension and its gel + grape seed extract $0.5 \%(\mathrm{w} / \mathrm{w})$ \\
TC & tendon suspension and its gel \\
TCP003 & tendon suspension and its gel + grape seed extract $0.03 \%(\mathrm{w} / \mathrm{w})$ \\
TCP05 & tendon suspension and its gel + grape seed extract $0.5 \%(\mathrm{w} / \mathrm{w})$ \\
\hline
\end{tabular}

Table 1 - Scaffolds denomination

\section{Methods \\ Differential scanning calorimetry (DSC)}

DSC curves were obtained with a computer-interfaced differential scanning calorimeter (Model DSC 2010, TA Instruments, New Castle, DE, U.S.A.). Samples of $20 \mathrm{mg}$ were sealed in hermetic aluminum pans and equilibrated at the initial temperature. The scanning was carried out with a heating rate of $10^{\circ} \mathrm{C} \mathrm{min}^{-1}$ from 5 to $120^{\circ} \mathrm{C}$ and performed under a nitrogen atmosphere. Denaturation temperature (Td) was determined as the inflection point value of the corresponding endothermic effect.

\section{Scanning Electorn Microscopy (SEM)}

Samples were coated with a thin layer of gold of $6 \mathrm{~nm}$. The specimens were examined with a Leo 440, LEO Electrorn Microscopy Ltd. (Cambridge, England) with an accelerating voltage of $20 \mathrm{keV}$. For the cross section analysis, the scaffolds were frozen in liquid nitrogen, fractured and Iyophilized. To measure the pore size, ImageJ software was used and for each scaffold, 31 determinations were made using the approximation of the Martin's diameter and an image magnification of 500x. ${ }^{18}$

\section{Absorption kinetics in phosphate buffered saline (PBS)}

Scaffolds were placed in vials containing phosphate buffered saline (PBS). At specific time intervals, scaffolds were removed from the PBS and the excess of solution was removed using a $2 \mathrm{~cm} \times 2 \mathrm{~cm}$ filter paper. The scaffolds were weighed and returned to the vials for further time taken. The process was made in quintuplicate. The amount of PBS absorbed was calculated by averaging the results found using equation (1), where $w_{\text {humid }}$ is the mass of the swollen scaffold and $w_{\text {dry }}$ is the mass of the scaffold before swelling.

$$
\% \text { absorption }=\frac{\left(w_{\text {humid }}-w_{\text {dry }}\right)}{w_{d r y}} \times 100
$$

\section{In vitro biological stability (collagenase)}

For in vitro biological stability determination, collagenase solution in $10 \mathrm{mmol} \mathrm{L}^{-1}$ tris- $\mathrm{HCl}$ buffer at $\mathrm{pH} 7.4$ containing $25 \mathrm{mmol} \mathrm{L}^{-1} \mathrm{CaCl}_{2}$ was prepared. Collagenase solution (10 $\mathrm{U} \mathrm{mg}^{-1}$ of collagen) in tris- $\mathrm{HCl}$ buffer, was added to scaffolds known weights which were subsequently placed in a bacteriological oven at $37^{\circ} \mathrm{C}$ for $2 \mathrm{~h}$. After this, the samples were washed deionized water, frozen and lyophilized to constant weight. The percentage of degraded collagen (\% degradation) was determined by the difference in collagen mass before $\left(\mathrm{w}_{\text {initial }}\right)$ and after enzymatic degradation $\left(w_{\text {final }}\right)$ by equation (2), and at least three determinations were made for each scaffold.

$$
\% \text { degradation }=\frac{\left(w_{\text {initial }}-w_{\text {final }}\right)}{w_{\text {initial }}} \times 100
$$

The analysis results were analyzed using ANOVA. A value of $p<0.05$ was considered statistically significant.

\section{Results and discussion}

The presence of grape seed extract is noted for its beige color in TCP003 and SCP003 scaffolds and brown in TCP05 and SCP05 scaffolds. The scaffolds without extract (TC and SC) are white and all of them presented touch-resistant appearance and reversibility to small deformations.

DSC curves showed a transition that appears as a discontinuity at baseline, giving the collagen denaturation temperature (Td). Denaturation occurs due to heating and can be defined as the transition of the collagen triple helix to a form in which intramolecular bonds are broken down, changing from a highly organized structure to a disorganized state called gelatin. ${ }^{19}$ The values of collagen denaturation temperatures are shown in Table 2.

Native tissues (tendon and serosa) had a denaturation temperature of $63.1^{\circ} \mathrm{C}$, values close to those found by Willett ${ }^{20}$ and Hizaji ${ }^{21}$, who analyzed the Td of bovine tail tendon collagen and obtained values of $62.4^{\circ} \mathrm{C}$ and $63.3^{\circ} \mathrm{C}$, respectively. Hirata 22 evaluated the Td of bovine serosa and obtained a value of $64.6^{\circ} \mathrm{C}$, close to those found in this study.

Therefore, a reduction in $\mathrm{Td}$ of 9.8 and $3.2^{\circ} \mathrm{C}$ is observed for native tissues compared to their respective TC and SC scaffolds, respectively. This indicates that alkaline treatment destabilizes the triple collagen helix, and that this destabilizing effect is greatest in tendon matrices.

Alkaline hydrolysis also modifies the macromolecular aggregation of native collagen ${ }^{23}$, which is different for tendon and serosa tissue. ${ }^{7}$ Differences in Td of SC and TC may indicate that the structure of the serosa is more resistant to destabilization, keeping part of its intrinsically crosslinked loose connective tissue structure.

The denaturation temperature of TC and TCP003 are very close, as well as for SC e SCP003 suggesting that crosslinking by grape seed extract was not effective at $0.03 \%$ concentration.

Scaffolds containing $0.5 \%$ of extract had an increase in denaturation temperature when compared to the scaffolds without extract, being of $14.7^{\circ} \mathrm{C}$ for the bovine tendon scaffolds and $6.4^{\circ} \mathrm{C}$ for the serosa scaffolds, indicating that in both cases $0.5 \%$ of extract is effective as a crosslinking agent.

In addition, the $0.5 \%$ extract cross-links collagen to the extent that the Td of SCP05 and TCP05 is larger than that of their respective originating tissues. This is because there are still crosslink available sites in the tendon and serosa and the proanthocyanidin present in the extract binds to available sites of the aminoacid proline group present in collagen, causing crosslinking. ${ }^{24}$

Scanning electronic microscopy allows to define the morphology of the scaffolds microstructures, such as pore size, distribution, porosity and interconnectivity, which are major factors in the interaction of the scaffolds with physiological environment, promoting or not the absorption of physiological liquids.

\section{Statistical Analysis}


Garcia et al.

\begin{tabular}{cc}
\hline Scaffold & Td $\left({ }^{\circ} \mathrm{C}\right)$ \\
\hline Bovine tendon & 63.1 \\
Porcine serosa & 63.1 \\
TC & 53.3 \\
SC & 59.9 \\
TCP003 & 53.7 \\
SCP003 & 60.8 \\
TCP05 & 68.0 \\
SCP05 & 66.3 \\
\hline
\end{tabular}

Table 2 - Denaturation temperature (Td).

Photomicrographs (Figures 1-6) show the presence of surface pores and in the cross-section for all samples. Table 3 shows the average pore size, with the respective standard deviations (sd) and the coefficient of variation (Cv).

No statistical difference was observed between TC and TCP05 pore sizes, indicating that the extract addition is not a major factor in pore size in bovine tendon scaffolds. However, for serosa scaffolds, the addition of extract at concentrations of $0.03 \%$ and $0.5 \%$ causes the emergence of pores pores on the scaffolds surface.

The histograms show that TC scaffold (Figure 2), with $74.0 \% \mathrm{Cv}$, has a bimodal system with a pore size frequency between 10-20 $\mu \mathrm{m}$ and 30-40 $\mu \mathrm{m}$. In the case of the SCPO03 scaffold (Figure 3), it also has a bimodal system with higher pore size frequencies between 20-30 $\mu \mathrm{m}$ and 30-40 $\mu \mathrm{m}$. The other scaffolds have systems with one mode, and for the scaffolds TCPO03 (Figure 4), SCP05 (Figure 5) and TCP05 (Figure 6) the largest frequency of pore size occurs between $40-50 \mu \mathrm{m}, 20-30$ $\mu \mathrm{m}$, and $30-40 \mu \mathrm{m}$, respectively. In the cross section, it is observed that only the TCP003 scaffold has a bimodal system, with a higher incidence of pores between 40-50 $\mu \mathrm{m}$ and 50-60 $\mu \mathrm{m}$. Only two scaffolds showed statistical difference between the pore size of the surface and the cross section. SC scaffold showed no pores on the surface and SCP05, which presented great pore variation in its cross section, presenting $\mathrm{Cv}$ of $54.2 \%$.

Nyberg $^{25}$ (2017) and Zhang ${ }^{26}$ (2018) show that pores smaller than 100 $\mu \mathrm{m}$ facilitate the diffusion of physiological fluids within the scaffold and promote a capillary force that improves cell attachment in the periphery of the scaffold and can induce cell penetration.

The ability of a scaffold to retain water and electrolytes is an important property for evaluating its effectiveness as a hemostatic material, so the absorption test is essential. Absorption of saline phosphate buffer by collagen scaffolds arises due to various electrostatic interactions. When the first water molecules enter the pores of the scaffold, they bind (hydrate) the hydrophilic groups present in collagen and grape seed extract. The
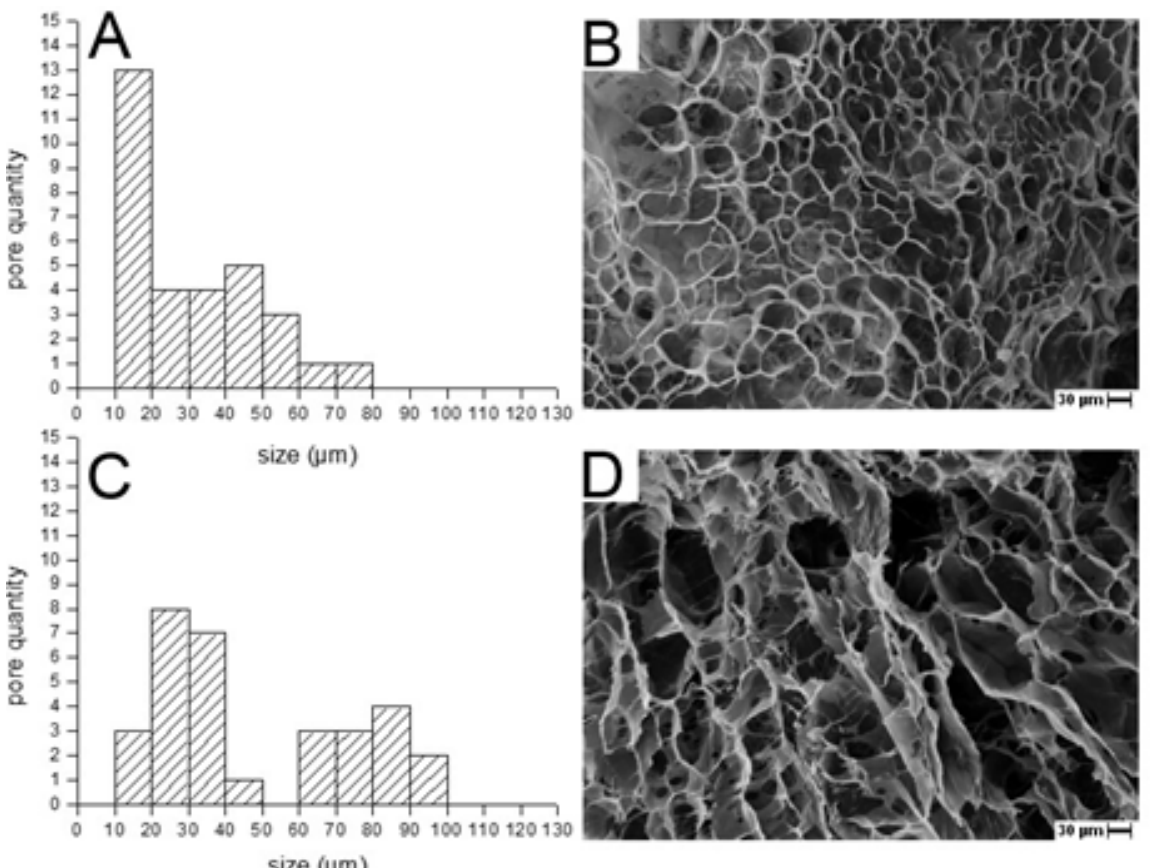

Figure 1 - SC scaffold: (A) surface pore size distribution histogram; (B) surface photomicrograph; (C) cross-sectional pore size histogram (D) cross section photomicrograph. 
Effects of grape seed extract on...
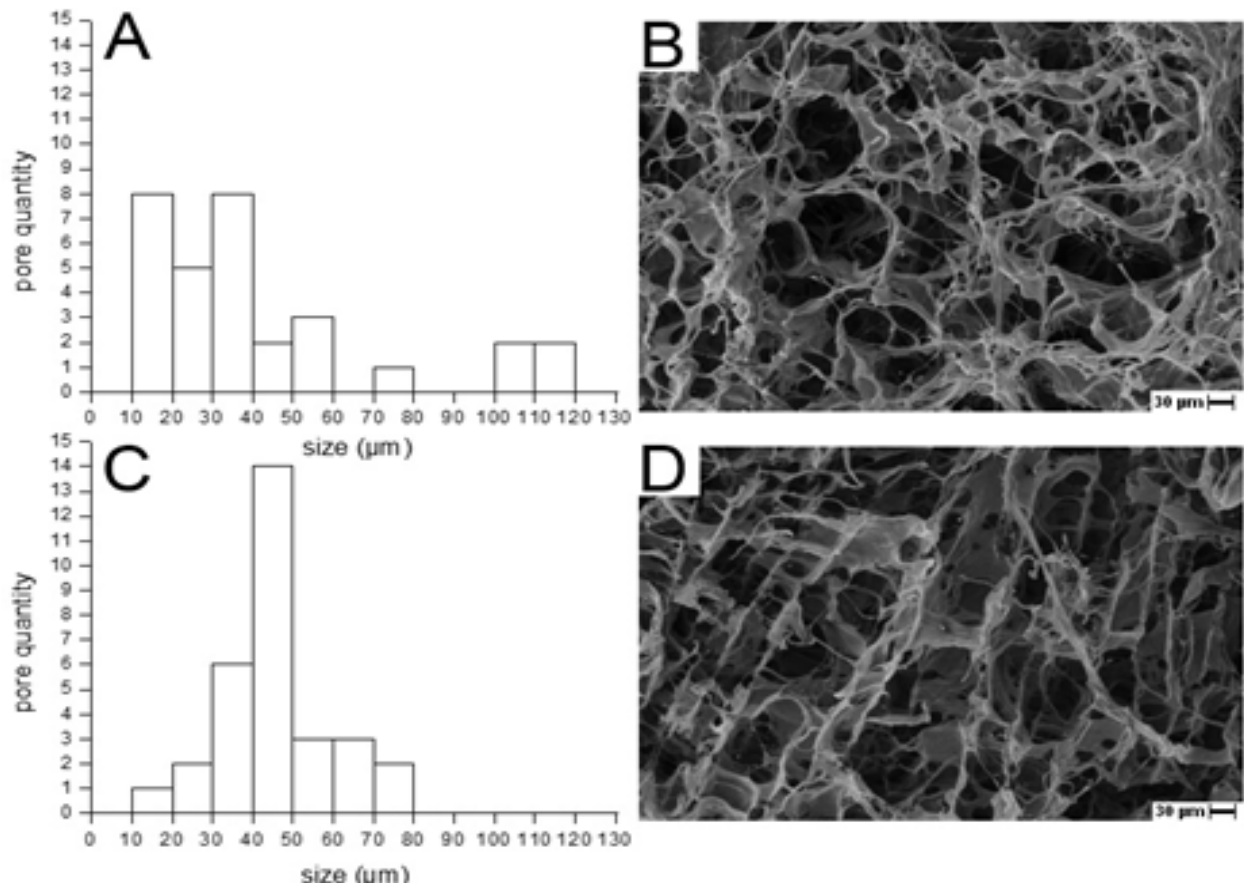

Figure 2 - TC scaffold: (A) surface pore size distribution histogram; (B) surface photomicrograph; (C) cross-sectional pore size histogram (D) cross section photomicrograph.
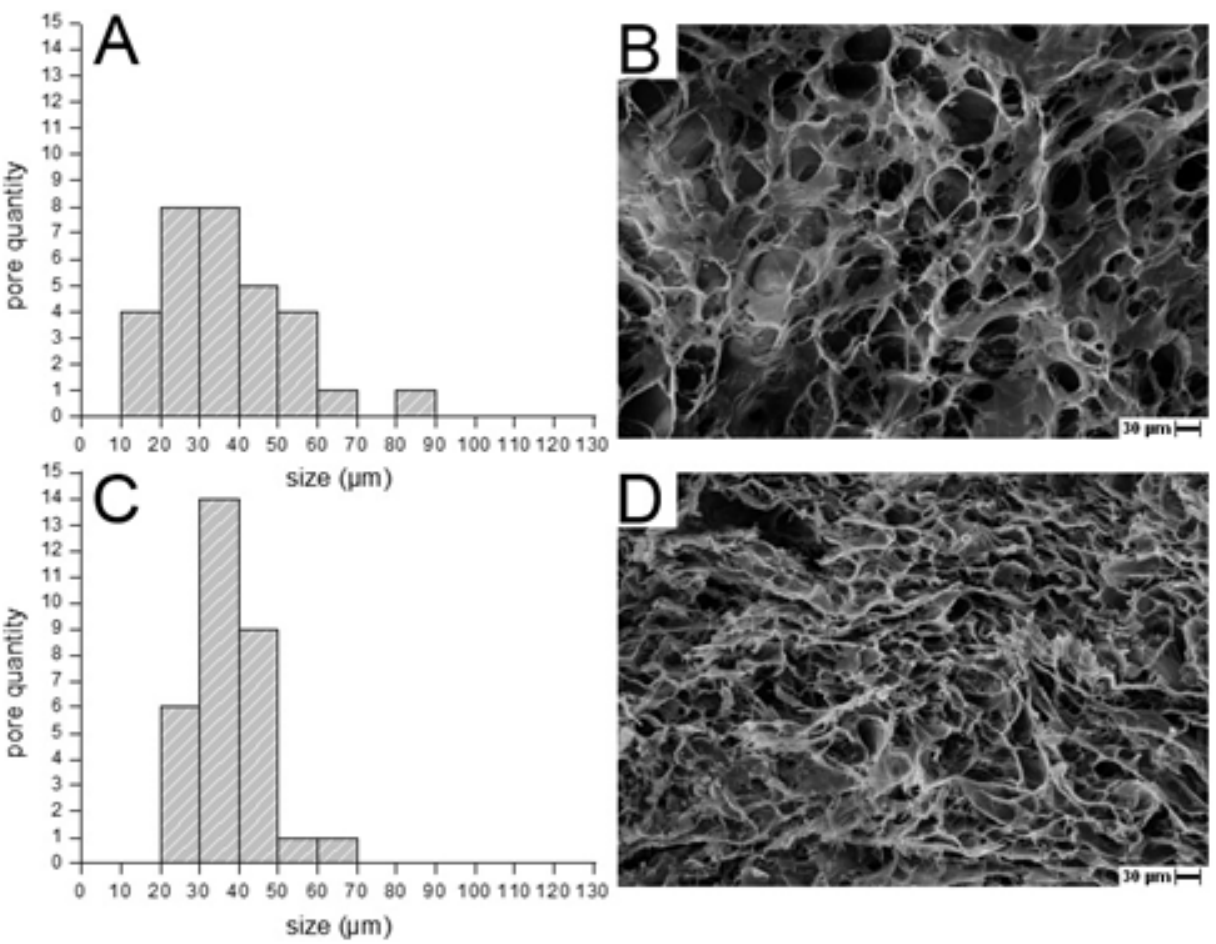

Figure 3 - SCP003 scaffold: (A) surface pore size histogram; (B) surface photomicrograph; (C) cross-sectional pore size histogram (D) cross section photomicrograph . 
Garcia et al.
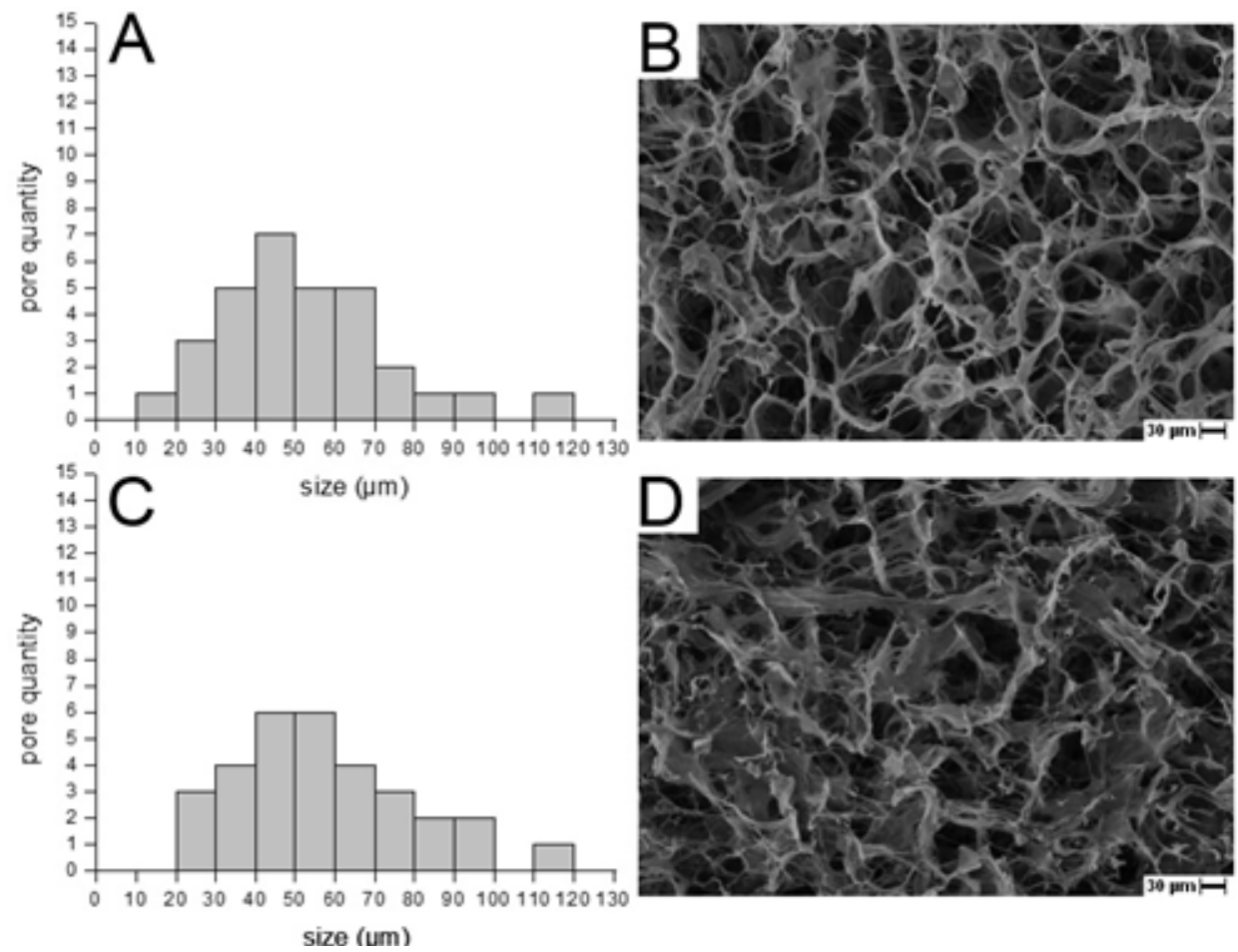

Figure 4 - TCP003 scaffold: (A) surface pore size distribution histogram; (B) surface photomicrograph; (C) cross-sectional pore size histogram (D) cross section photomicrograph.
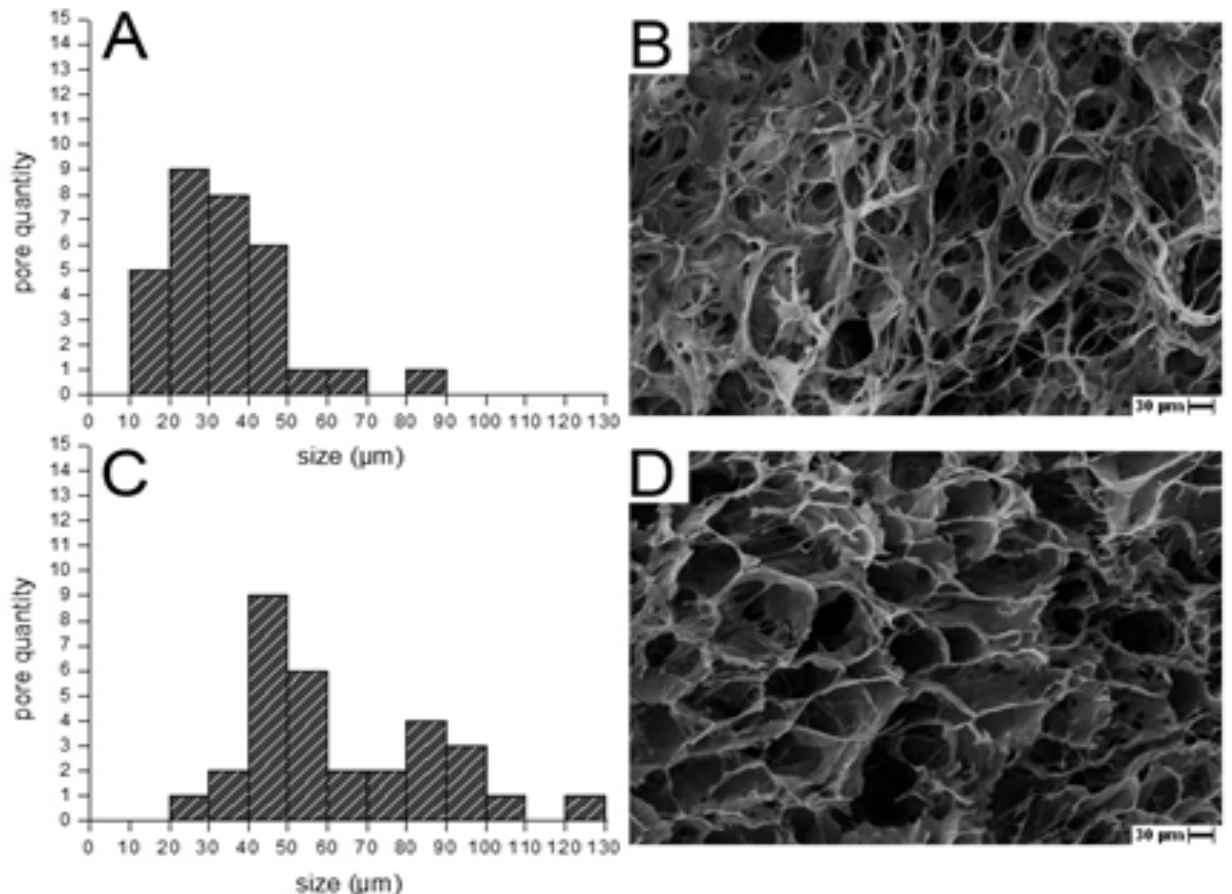

Figure 5 - SCP05 scaffold: (A) surface pore size histogram; (B) surface photomicrograph; (C) cross-sectional pore size histogram (D) cross section photomicrograph. 


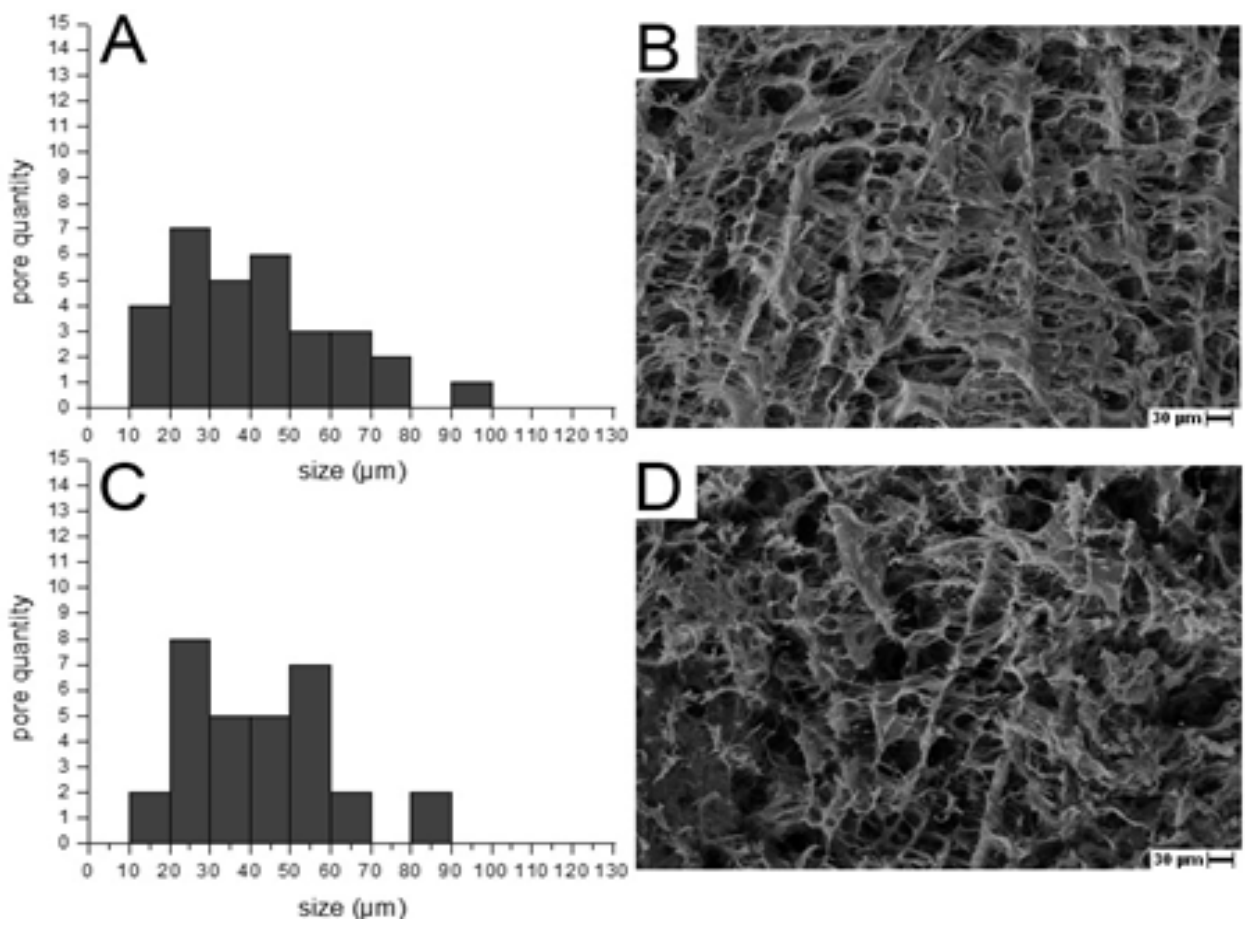

Figure 6 - TCP05 scaffold: (A) surface pore size distribution histogram; (B) surface photomicrograph; (C) cross-sectional pore size histogram (D) cross section photomicrograph.

\begin{tabular}{ccccc}
\hline \multirow{2}{*}{ Scaffolds } & \multicolumn{3}{c}{ Mean \pm sd $(\mu \mathrm{m})$} \\
\cline { 2 - 5 } & Surface & Cv $(\%)$ & Cross section & Cv $(\%)$ \\
\hline TC & $41.1 \pm 30.4^{\mathrm{a}, \mathrm{b}}$ & 74.0 & $43.2 \pm 13.7^{\mathrm{b}, \mathrm{d}}$ & 30.3 \\
SC & $31.1 \pm 17.8^{\mathrm{b}}$ & 57.1 & $48.4 \pm 26.2^{\mathrm{b}, \mathrm{c}}$ & 54.2 \\
TCP003 & $52.9 \pm 21.0^{\mathrm{a}}$ & 39.7 & $56.7 \pm 22.2^{\mathrm{a}, \mathrm{b}}$ & 39.2 \\
SCP003 & $36.4 \pm 16.6^{\mathrm{b}}$ & 45.5 & $38.3 \pm 9.3^{\mathrm{c}, \mathrm{d}}$ & 24.2 \\
TCP05 & $41.9 \pm 19.5^{\mathrm{a}, \mathrm{b}}$ & 46.4 & $41.2 \pm 17.5^{\mathrm{c}, \mathrm{d}}$ & 42.5 \\
SCP05 & $32.4 \pm 14.5^{\mathrm{b}}$ & 44.6 & $63.1 \pm 23.8^{\mathrm{a}}$ & 37.7 \\
\hline
\end{tabular}

In the same column, same letter means statistically equal numbers $(p<0.05)$ sd: standard deviations. Cv: coefficient of variation $(\%)$.

Table 3 - Surface and cross section pore size of the scaffolds.

adhesive interactions between the various polar aminoacids residues present in collagen and PBS are stronger than the cohesive forces of the buffer itself. This causes the buffer to be absorbed throughout the scaffold, causing it to swell. PBS absorption at 30,60 and $1200 \mathrm{~min}$ is shown at Table 4.

Figure 7 shows the percentage of absorption at different times. TCP05 and TCP003 had their maximum absorption at $2750 \%$ in the first minute, different of TC, which had maximum absorption at $1920 \%$ after 1200 minutes.

Serosa scaffolds absorbed their maximum around 30 minutes, which SC and SCP003 had statistically equal absorption around $1400 \%$ and the SCP05 absorbed around $2750 \%$.

Scaffolds TCP05 and SCP05 have statistically equal absorption around $2700 \%$, almost 2 times higher than the scaffolds without extract. This is due to the large number of hydroxyls groups present in proanthocyanidin, which make the material most hydrophilic. ${ }^{27}$

Swelling of a material is hampered by inter and intramolecular bonding, intrinsic stiffness and degree of crosslinking. ${ }^{28}$ Then, a difference in the absorption of the TC and SC was expected, since the serosa scaffold showed higher thermal stability, which may be due to a larger number of bonds in its structure (intrinsic cross-linking).

This difference is only observed between TCP003 and SCP003, since the presence of $0.03 \%$ extract is not able to crosslink collagen to the point of increasing its $\mathrm{Td}$, but it contributes to the hydrophilicity of the material, making the scaffold TCP003 more hydrophilic than SCP003, which is difficult to absorb due to its intrinsic cross-linking.

\section{In vitro biological stability (collagenase)}

Collagenase biological stability assays were designed as a relative indication of the biodegradability of post implant scaffolds. In vitro degradation of collagen by collagenase occurs in three stages: i) they are diffusion, in which collagenase in the solution diffuses through the substrate. In this case, it is collagen itself; ii) absorption, in which the enzyme is adsorbed onto the collagen surface and, iii) degradation, in which the molecule is broken into minors fragments. ${ }^{29}$

Since scaffolds, TCP003 and SCP003, did not show an effective cross- 


\section{Garcia et al.}

linking as observed by their denaturation temperature, only TC, SC, TCP05 and SCP05 were used in the degradation assays, shown in Table 5.

The scaffold TC presented the higher percentage of degradation, being $74.0 \pm 5.0$ and, therefore, having lower biological stability in vitro. The scaffold SC presented a degradation percentage of $9.3 \pm 2.1$, indicating greater stability of serosa collagen against collagenase, probably due to the higher number of intrinsic serosa tissue crosslinks, as already mentioned.

With the addition of $0.5 \%$ extract, the percentage of tendon scaffolds degradation decreased from $74.0 \pm 5.0$ to $15.3 \pm 0.4$ due to crosslinking caused by proanthocyanidin and other flavonoids present in grape seed extract.

Degradation percentage for SC and SCP05 were expected to be statistically different, however this was not observed. Maybe another test using a higher degradation time could result in a difference of degradation between serosa scaffolds.

$\mathrm{Ma}^{30}$ evaluated the biodegradability of collagen of $0.25 \%$ glutaraldehyde crosslinked bovine tendon observing that there was a reduction of biodegradability of approximately $87 \%$, close to the observed in this study with tendon scaffold that when cross-linked with $0.5 \%$ extract showed reduction of approximately $80 \%$.

Rodrigues $^{31}$ also evaluated trypsin enzymatic stability of glutaraldehyde cross-linked collagen. Glutaraldehyde cross-linking reduces the degradation percentage of collagen scaffolds too. This indicates a possible substitution of glutaraldehyde for the extract, as glutaraldehyde presents high cytotoxicity. ${ }^{32}$

\begin{tabular}{cccc}
\hline \multirow{2}{*}{ Scaffold } & \multicolumn{3}{c}{ Absorption (\%) \pm sd } \\
\cline { 2 - 4 } & $\mathbf{3 0} \mathbf{m i n}$ & $\mathbf{6 0} \mathbf{~ m i n}$ & $\mathbf{1 2 0 0} \mathbf{~ m i n}$ \\
\hline TC & $1575 \pm 217^{\mathrm{b}}$ & $1753 \pm 239^{\mathrm{b}}$ & $1921 \pm 188^{\mathrm{b}}$ \\
SC & $1374 \pm 131^{\mathrm{b}}$ & $1397 \pm 124^{\mathrm{b}}$ & $1489 \pm 152^{\mathrm{b}}$ \\
TCP003 & $2739 \pm 192^{\mathrm{a}}$ & $2791 \pm 179^{\mathrm{a}}$ & $2872 \pm 186^{\mathrm{a}}$ \\
SCP003 & $1469 \pm 106^{\mathrm{b}}$ & $1511 \pm 087^{\mathrm{b}}$ & $1579 \pm 051^{\mathrm{b}}$ \\
TCP05 & $2342 \pm 305^{\mathrm{a}}$ & $2531 \pm 205^{\mathrm{a}}$ & $2572 \pm 214^{\mathrm{a}}$ \\
SCP05 & $2813 \pm 580^{\mathrm{a}}$ & $2855 \pm 524^{\mathrm{a}}$ & $2806 \pm 519^{\mathrm{a}}$ \\
\hline
\end{tabular}

In the same column, same letter means statistically equal numbers $(p<0.05)$ sd: standard deviations.

Table 4 - PBS absorption kinetics (\%).

\begin{tabular}{cc}
\hline Scaffold & Degradation (\%) \pm sd \\
\hline TC & $74.0 \pm 5.0^{\mathrm{a}}$ \\
SC & $9.3 \pm 2.1^{\mathrm{b}, \mathrm{c}}$ \\
TCP05 & $15.3 \pm 0.4^{\mathrm{b}}$ \\
SCP05 & $3.7 \pm 2.5^{\mathrm{c}}$ \\
\hline
\end{tabular}

In the table, same letter means statistically equal numbers $(p<0.05)$ sd: standard deviations.

Table 5 - Scaffolds degradation \pm sd. 


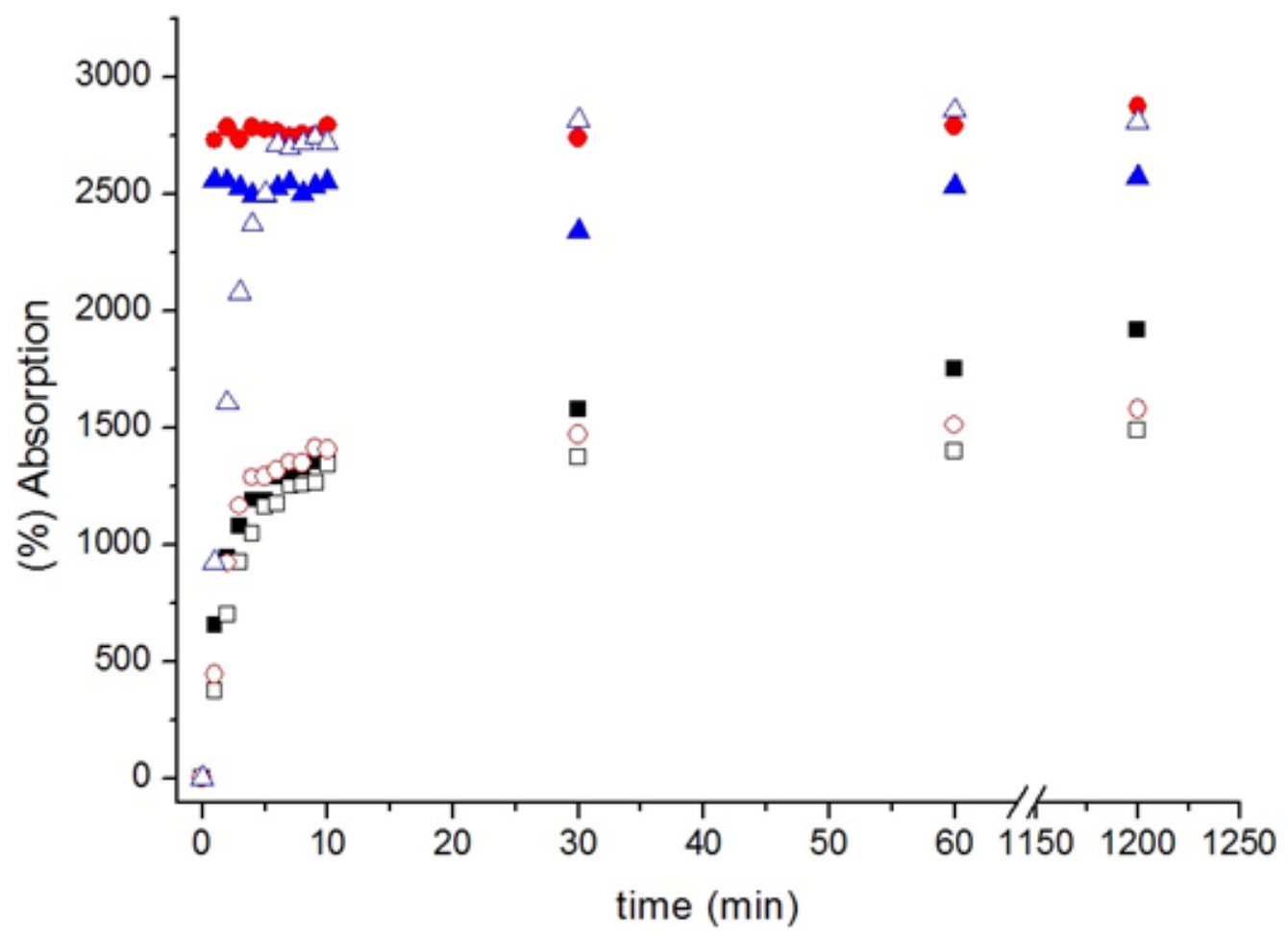

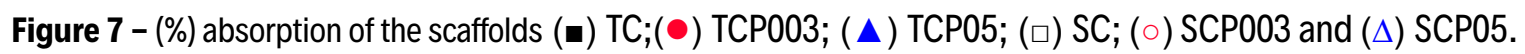

\section{Conclusion}

Photomicrographs showed the presence of surface pores and in the cross-section for all samples, not larger than $130 \mu \mathrm{m}$. Cross-linking by $0.5 \%$ extract increases collagen denaturation temperature, reduces degradation percentage 12 and increases the swelling, which may result in scaffolds for different applications such as hemostatic agent or soft tissue regeneration.

\section{Conflict Of Interests}

There are no conflicts to declare.

\section{Acnlowledgements}

The authors would like to thank the Center of Analytical Chemical Analysis of IQSC/USP, for all the infrastructure available for SEM analysis.

\section{Funding}

This study was supported by São Paulo Research Foundation (FAPESP), process number 2015/15473-7, and National Council for Scientific and Technological Development (CNPq) contract number 130686/2018-2.

\section{References}

1. Tomizawa Y. Clinical benefits and risk analysis of topical hemostats: a review. J of Artificial Organs 8(3) : 137-142 (2005).

2. Carvalho M V H, Marchi E, Pantoroto M, Rossini M, Silva DMS, Teodoro LFF, Pantaroto A. Agentes hemostáticos locais e adesivos teciduais. Revista do Colégio Brasileiro de Cirurgiões 40(1) : 66-71 (2013).

3. Barnard J, Millner R. A Review of Topical Hemostatic Agents for Use in Cardiac Surgery. Annals of Thoracic. Surgery 88 : 1377-1383 (2009).

4. DeFrates KG, Moore R, Borgesi J, Lin G, Mulderig T, Beachley V, Hu $X$, Protein-Based Fiber Materials in Medicine: A Review. Nanomaterials 8457 (2018).
5. Hess JR, Brohi K, Dutton RP, Hauser CJ, Holcomb JB, Kluger Y, The coagulopathy of trauma: a review of mechanisms. J Trauma 65 : 748-754 (2008).

6. Sundaram $\mathrm{CP}$, Keenan $\mathrm{AC}$ Evolution of hemostatic agents in surgical practice. Indian J Urol. 26(3) : 374-78 (2010).

7. Junqueira LC, Carneiro J, Histologia Básica. ed 12. - Rio de Janeiro: Guanabara Koogan (2013).

8. Willett T L, Dapaah DY, Uppuganti S, Granke M, Nymanb JS. Bone collagen network integrity and transverse fracture toughness of human cortical bone. Bone, 120 : 187-193 (2019).

9. Sun J, Mou C, Shi Q, Chen B, Hou X, Zhang W, Li X, Zhuang Y, Shi J, Chen Y, Dai J. Controlled release of collagen-binding SFF-1 $\square$ from collagen scaffold promoted tendon regeneration in a rat Achilles tendon defect model. Biomaterials, 162 : 22-33 (2018).

10. Bet MR, Goissis G, Lacerda CA. Characterization of polyanionic collagen prepared by selective hydrolysis of asparagine and glutamine carboxyamide side chains. Biomacromolecules, 2(4) : 10741079 (2001).

11. Goissis G, Suzigan S, Parreira DR, Maniglia JV, Braile DM, Raymundo SRO. Preparation and characterization of collagen-elastin matrices from blood vessels intended as small diameter vascular grafts. Artificial Organs, 24(3) : 217-223 (2000).

12. Rocha LB, Goissis G, Ross MA. Biocompatibility of Anionic Collagen Matrix as Scaffold for Boe Healing. Biomaterials, 23 : 449-456 (2002).

13. Forti FL, Goissis G, Plepis AMG. Modifications on Collagen Structures Promoted by 1,4-Dioxane Improve Thermal and Biological Properties of Bovine Pericardium as a Biomaterial. J of Biomaterials Applications, 20 : 267-285 (2006). 
14. Chen Q, Liang S, Thouas GA. Elastomeric biomaterials for tissue engineering. Progress in Polymer Science, 38 : 584 - 671 (2013).

15. Han B, Jaurequi J, Tang BW, Nimni ME. Proanthocyanidin: A natural crosslinking reagent for stabilizing collagen matrices. J Biomedical Materials, 65 : 118-124 (2003).

16. Cho ML, Heo YJ, Park MK, Oh HJ, Park JS, Woo YJ, Ju JH. Grape seed proanthocyanidin extract (GSPE) attenuates collagen-induced arthritis. Immunology Letters, 124(2) : 102-110 (2009).

17. Horn MM, Martins VCA, Plepis AMG. Interaction of anionic collagen with chitosan: Effect on thermal and morphological characteristics. Carbohydrate Polymers, 77(2) : 239-243 (2009).

18. Allen, T. Particle Size Measurement- Powder sampling and particle size measurement. 5 ed, Londres, Chapman \& Hall, pp 525 (1997).

19. Parenteau-Bareil R, Gauvin R, Berthod F. Collagen-Based Biomaterials for Tissue Engineering Applications. Materials, 3(3) : 1863-1887 (2010).

20. Willett TL, Labow RS, Aldous IG, Avery NC, Lee JM. Changes in collagen with aging maintain molecular stability after overload: Evidence from an in vitro tendon model. Journal of Biomechanical Engineering, 132(3) (2010).

21. Hijazi KM, Singfield KL, Veres SP. Ultrastructural response of tendon to excessive level or duration of tensile load supports that collagen fibrils are mechanically continuous. Journal of the Mechanical Behavior of Biomedical Materials, 97 : 30-40 (2019).

22. Hirata HH, Munhoz MAS, Plepis AMG, Martins VCA, Santos GR, Galdeano EA, Cunha MR. Feasibility study of collagen membranes derived from bovine pericardium and intestinal serosa for the repair of cranial defects in ovariectomised rats. Injury, 46(7) : 1215-1222 (2015).

23. Lacerda C, Plepis AMG, Goissis G. Hidrólise seletiva de carboxiamidas de resíduos de asparagina e glutamina em colágeno: preparação e caracterização de matrizes aniônicas para uso como biomateriais, Quím Nova, 21(3) : 267-271 (1998)

24. Vidal CMP, Zhu W, Manohar S, Aydin B, Keiderling TA, Messersmith PB, Bedran-Russo AK. Collagen-collagen interactions mediated by plant-derived proanthocyanidins: A spectroscopic and atomic force microscopy study. Acta Biomaterialia, 41 : 110-118 (2016).

25. Nyberg E, Rindone A, Dorafshar A, Grayson WL. Comparison of 3D-Printed Poly-e-Caprolactone Scaffolds Functionalized with Tricalcium Phosphate, Hydroxyapatite, Bio-Oss, or Decellularized Bone Matrix. Tissue Engineering, 23 : 503-514 (2017).

26. Zhang K, Fan Y, Dunne N, Li X. Effect of microporosity on scaffolds for bone tissue enginnering. Regenerative Biomaterials 5 : 115124 (2018).

27. Sionkowska A, Kaczmarek B, Lewandowska K. Modification of collagen and chitosan mixtures by the addition of tannic acid. $J$ of Molecular Liquids, 199 : 318-323 (2014).

28. Walters BD, Stegemann JP. Strategies for directing the structure and function of three-dimensional collagen biomaterials across length scales. Acta Biomaterialia, 10 : 1488-1501 (2014).

29. Metzmacher I. Enzymatic degradation and drug release behavior of dense collagen implants. Dissertation - Department of Pharmacy, Pharmacetical Technology and Biopharmaceutics, Ludwig-Maximilians, University Munique, 2005.
30. Ma L, Gao C, Mao Z, Zhou J, Shen J, Hu X, Han C. Collagen/chitosan porous scaffolds with improved biostability for skin tissue engineering. Biomaterials, 24 : 4833-4841 (2003).

31. Rodrigues FT, Martins VCA, Plepis AMG. Porcine skin as a sources of biodegradable matrices: Alkaline treatment and glutaraldehyde crosslinking. Polímeros, 20(2) : 92-97 (2010).

32. Takigawa T, Endo Y. Effects of glutaraldehyde exposure on human health. J of Occupational Health, 48 : 75-87. (2006). 